\title{
Taming the Heathkit-Votrax speech synthesizer
}

\author{
PHILLIP L. EMERSON, DORIS C. KARNISKY, and CARLA J. KASTANIS \\ Cleveland State University, Cleveland, Ohio
}

\begin{abstract}
Three inexpensive text-to-speech synthesizers are described, intelligibility data from a pilot experiment are reported, and software is offered that has been written to facilitate the phonemic programming of the Heathkit-Votrax synthesizer.
\end{abstract}

Our main contribution is a set of IBM PC programs designed to ease the task of finding intelligible phoneme sequences to substitute for English word spellings in programming the Heathkit HV2000 speech synthesizer, which is based on the Votrax SCO2 IC chip. However, it would be hard to understand why such software is important and useful, without a bit of background in the rapidly developing area of computer speech processing. Therefore, we will begin with some distinctions and facts concerning the art of speech processing on personal computers. Then we will briefly describe some experimental data that we have collected, concerning the intelligibilities of three inexpensive synthesizers, including the Heathkit-Votrax. Our experiences in conducting those experiments led us to realize the need for software of the kind that we are now making available to other scientific and educational users.

\section{Speech Processing on Small Computers}

A large tree diagram might be the best way to organize all that is known about speech processing on small computers. We mention this large tree only to keep our own modest twig of a contribution in perspective; there are many large and interesting limbs that cannot be explored here. The first big fork occurs between computer recognition and computer production of speech. Our software is concerned with speech production, and up that branch we find that the next main fork has three limbs. These are three different methods, which can be ordered along a dimension that consists of the amounts of high-speed primary memory required. They are: (1) pulse code modulation, or PCM; (2) linear predictive coding, or LPC; and (3) formant coding, or FC. This nomenclature is not entirely self-explanatory, but it has become rather standard.

PCM is the straightforward method in which a sound wave is represented as a digitized recording of amplitude numbers taken at equally spaced time intervals. As a general rule, the Nyquist requirement for intelligible speech is about 8,000 PCM samples per second. Thus, if each amplitude is encoded as an 8-bit number (which is often found adequate, as well as convenient), a $64 \mathrm{~K}$

Correspondence should be addressed to Phillip L. Emerson, Cleveland State University, Cleveland, OH $\mathbf{4 4 1 1 5 .}$ segment of memory is required to produce an 8-sec stretch of PCM speech. The computer produces the sound by putting the amplitude numbers through a D/A converter at equally spaced time intervals, to an audio amplifier and speaker. A personal computer CPU can make 8,000 D/A conversions per second, but it has little time left over to do other things concurrently. The $64 \mathrm{~K}$ segment is mentioned because the INTEL $8088 / 86$ computer design makes it easy to address $64 \mathrm{~K}$ of data memory, but it is a bit of a programming nuisance to address more. Popular versions of $C$ and Pascal for MS-DOS have the capability to address more than $64 \mathrm{~K}$, but some versions of BASIC do not.

LPC takes advantage of commonly occurring stretches of the acoustic wave of natural speech, in which one PCM sample is very closely predictable from the preceding one or from a short preceding series. Thus, a predictive algorithm can be invoked, sometimes implemented as a slave CPU programmed with ROM, to fill in with the needed PCM outputs, thereby leaving the central CPU freer to do other chores. The central CPU is not in such a tight timing loop, and its primary memory representation of the sound wave can be much more compact. The memory representation is a record, in some form, of occasional changes that need to be made in the output sound wave, rather than a detailed record of the sound wave itself.

FC is a more direct attempt to utilize findings from research on natural speech. There are several broad bands of frequencies observable in speech spectrograms that have a lot to do with how speech sounds are perceived. They are called formants, and each may change in center frequency for different vowel sounds. Such changes tend to be fairly smooth, except at boundaries between voiced and unvoiced speech segments. The formants tend to disappear for nonvoiced consonants, which look more like a splattering in the spectrogram than a set of bands, but the formant transitions between voiced and unvoiced segments are important even for the perception of unvoiced phonemes. Before small computers were available for speech processing, speech was synthesized by an FC method of sketching formants on a chart, which was then fed to a device much like a player piano.

LPC and FC implementations are often based on mathematical models of the acoustical characteristics of the vo- 
cal system. The goals of LPC and FC are to produce acceptable speech from a much smaller memory representation than is needed with PCM, and with lower data transfer rates. The catch is that most inexpensive implementations do not sound very natural, and that sometimes they are not very intelligible, whereas PCM sounds essentially the same as the natural voice that produced the sound that has been recorded.

\section{The Three Inexpensive Synthesizers}

The Appendix gives characteristics of the three synthesizers that were available to us for testing on an IBM PC. The main purpose of the Heathkit and B. G. Micro products is to have the computer produce artificial speech from ASCII text. That function is included with the COVOX product, but as just one option. The COVOX system also includes a PCM mode, which is used with some of the supplied software to do experimental editing of soundwaves. We have not yet tried to use the COVOX system in the PCM mode, because so far we have been focusing on the production of speech from text.

Each synthesizer uses its own set of rules for the textto-sound conversions. These rules are built into the synthesizers and are not specified explicitly in the user documentation. However, it is known from other sources that FC is the method of the Votrax IC used by Heathkit, and that LPC is the method of the Smoothtalker software (from First Byte, Inc.) used by COVOX. It is quite evident from listening to each synthesizer that the set of rules is a crude subset of those that would be needed to produce high-quality speech. For this reason, each synthesizer comes with facilities for using an auxiliary software dictionary file (that the user must create) containing alternate spellings of words or phrases that art mispronounced by the built-in rules. Before conducting the experiment to be described, the experimenters worked for a number of hours to construct such a lookup file for each synthesizer, repeatedly trying different spellings to get a set of common words to sound acceptable to our ears. This turned out to be a very tedious and fatiguing task, requiring much retyping of almost the same strings while trying to rememeber the sounds produced by the various retypings. This is the task for which we developed the new software to be described below.

\section{The Experiment}

This experiment is described quite briefly, because it was little more than a pilot study to teach us more about the needed methodology than about the synthesizers per se. Each of three groups of 10 subjects was tested for the correct recognition of 99 words (English versions of Swadesh's universal concepts; Miller, 1981), with one of the three synthesizers. Each word was presented in isolation on a single trial, in which the listener was allowed up to two repetitions of the word but was penalized in the scoring for each repetition. The test for overall differences among the synthesizers was not significant at the .05 level. However, the word main effects were highly significant, and the word $\times$ synthesizer interaction was very significant. Thus, across synthesizers, some words were consistently intelligible and some unintelligible, and other words were quite intelligible with one synthesizer but not with another. The Venn diagrams of Figures 1 and 2 show the best and worst words, for each synthesizer, and the overlapping subsets.

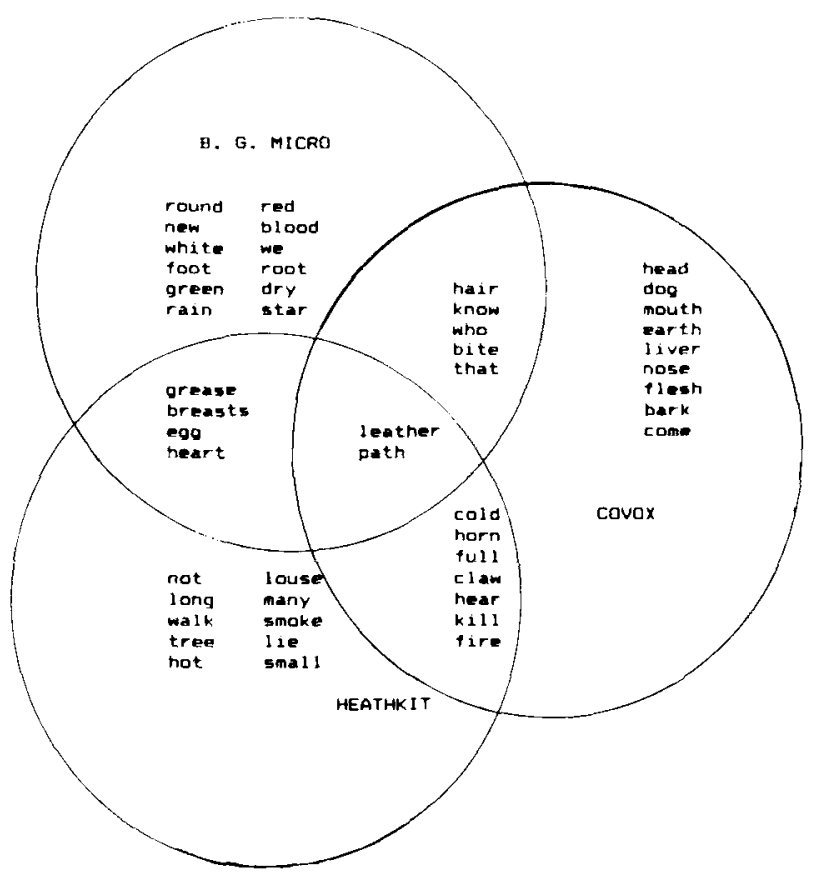

Figure 1. Words with average intelligibility scores below $17 \%$, for three synthesizers.

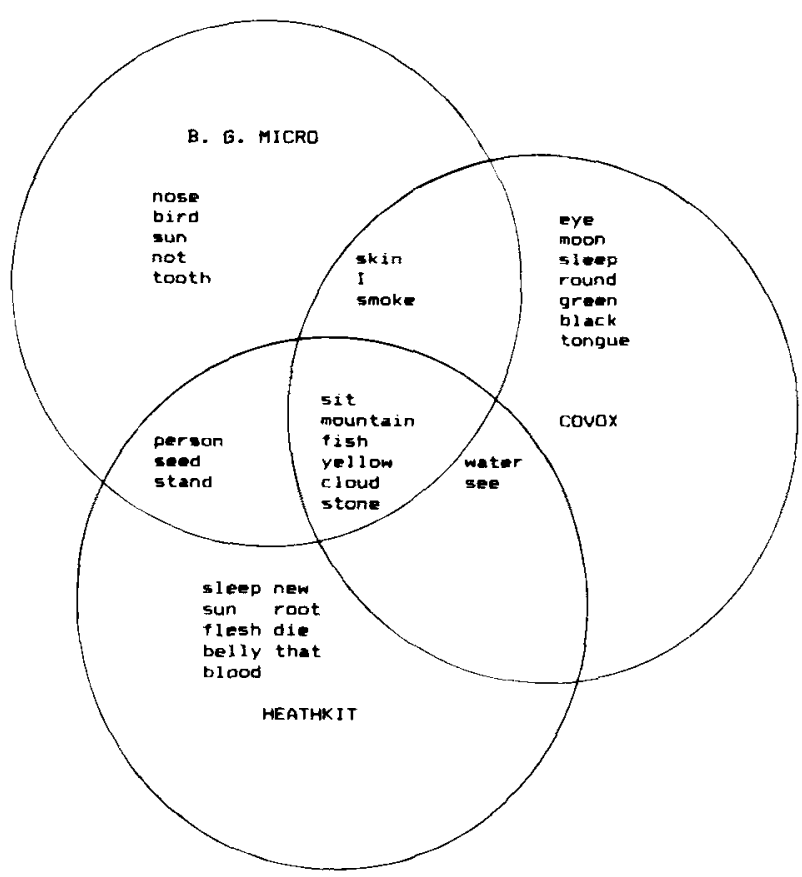

Figure 2. Words with average intelligibility scores above $83 \%$, for three synthesizers. 
The grand mean of intelligibility scores averaged over synthesizers, words, and subjects was about $43 \%$. This may seem too low for any useful application, but it is really not as bad as it may seem. In this experiment, each word was presented in isolation, and it is well known that intelligibility increases markedly when words are presented in natural linguistic context. Also, we purposely used naive subjects, though it is clear that there is improvement with practice in listening to a given synthesizer. Very likely there is also improvement with practice in the task that the experimenters performed in creating the phonemic dictionary files. None of us had much practice in this task before embarking on that project.

\section{The New Software}

Our data suggest a direction for further efforts to improve the dictionary file for each synthesizer. However, we were bothered very much by the possibility that the dictionary might not have been really optimized for each synthesizer, and that the overall intelligibility estimates and the test for synthesizer differences should therefore not be trusted very far. For that reason, new software was written to make it easier and faster to create and edit good dictionary files.

The present versions of these programs are designed to produce dictionary files only for the Heathkit synthesizer; they were by far the most difficult to produce, given the primitive methods provided by the vendor. One reason is that the Heathkit synthesizer provides more programming options than the others do. There are 64 phoneme symbols (including 30 allophones), 10 voiceattribute variables, and 6 global parameter options. In addition, as the Heathkit and Votrax documentation explains, some seemingly simple speech sounds are best produced by fairly long sequences of primitive phoneme symbols from the set of 64 . To make matters worse, some of the different parameter values must be combined and entered as a single hexadecimal number, while some single parameters must be split in a complicated way into two hexadecimal numbers.

To solve these annoying problems, it was decided that an interpretive program module was needed that would translate from a simplified format using decimal numbers with parameter values specifiable independently, into the required Heathkit-Votrax format using hexadecimal. Thus, a simplified language is used to formulate entries into the dictionary file, and the interpreter module (INTERP) converts to the format required for producing the sound. These two formats will be referred to here as SOD (SOurce Dictionary) and HED (HEathkit Dictionary) formats. There are two programs, both written in Turbo $\mathrm{C}$ (Version 1.5). One uses screen cursor addressing with an IBM or compatible personal computer. Their names are SODEDIT and SODHED.

SODEDIT is the primary program; it is a screen editor used to insert and modify items in a SOD dictionary file. While the user is trying different phonemic spellings of a word, playback is obtained whenever desired by the stroke of a certain key. Thus, the usual editing functions of BACKSPACE, DELETE, INSERT, OVERSTRIKE, and cursor movements are available for quickly changing the phoneme string, to be played back again while the sound from the previous playback is still fresh in mind. After a playback, the cursor remains where it was, so very little retyping and relocation are needed between playbacks while the user is working on some particular problematic segment of a word.

One dictionary item is created or edited at a time, using only the top few lines of the screen. The rest of the screen is used for several optional displays that provide useful information during editing, such as a list of the 64 phonemes, some examples of diphthong programming, a list of the voice attributes, ranges of parameter values, and default values. The first display that comes onto the lower screen when SODEDIT is loaded, is a list of Alt and $\mathrm{Ctrl}$ keys that can be used to select other displays and take other actions such as ending a session. At the end of a session, the new version of the dictionary is stored in a new file in SOD format.

The second program, SODHED, produces a HED file from an existing SOD file, so that an applications program need not contain the interpretive module (INTERP) used by SODEDIT and SODHED. Another program module that is used by both SODEDIT and SODHED, which is also in some applications programs, is LEXPAK. It provides for hash coding and dynamic memory allocation in the management of the lexicon of dictionary entries, after the methods discussed by Kernighan and Ritchie (1988, pp. 136-145). The purpose of the LEXPAK routines is to provide for very fast memory retrieval, using only as much memory as is actually needed for the given dictionary file.

\section{Conclusion}

Using the new software, we have found dictionary editing to be almost a pleasure. Heathkit supplies a facility for building a dictionary file (WORDS.EXC); but the length is limited to 100 items, and the editing is much less convenient than it is with SODEDIT. The SOD files produced by SODEDIT, on the other hand, have no length limit other than that determined by the amount of memory available. A few thousand items should be handled easily, using the 640 or $500 \mathrm{~K}$ of memory on most PCs and compatibles purchased in recent years. The entries in the dictionary files for our applications have so far been English words and their phonemic translations. The same software could be used for special applications to produce dictionaries of phrases. SODEDIT, however, does not work properly with SOD strings longer than the 80-byte width of the video screen.

The hard /g/ as in big, as it is produced by the Heathkit software, sounds very much like a hiccup, but we have learned how to produce a quite natural-sounding rendition of this phoneme and of some others, using combina- 
tions from the 64-element Votrax set. We have had less success with the nasals, and we are coming to believe that the fault rests with the Votrax $\mathrm{SCO} 2$ itself. As produced by the Votrax chip, $/ \mathrm{m} /$ is very confusable with $/ \mathrm{v} /$, and $/ \mathbf{n} /$ is confusable with $/ 1 /$ in some contexts.

Softcopy of the source and executable programs will be supplied on request from scientific and educational users, on 5.25-in. 360K IBM format disk. A few applications notes and demonstration programs are also in- cluded on the disk. The request should be accompanied by a post-paid self-addressed mailer and a blank diskette.

\section{REFERENCES}

Kernighan, B. W., Ritchie, D. M. (1988). The C programming language (2nd ed.). Englewood Cliffs, NJ: Prentice-Hall.

Miller, G. A. (1981). Language and speech. San Francisco: W. H. Freeman.

\begin{tabular}{|c|c|c|c|}
\hline \multicolumn{4}{|c|}{$\begin{array}{c}\text { APPENDIX } \\
\text { Characteristics of Three Speech Synthesizers }\end{array}$} \\
\hline Source: & covox & Heathkit & B. G. Micro \\
\hline Product: & Speech Thing & HV2000 & Computalker \\
\hline Price: & $\$ 80$ & $\$ 90$ & $\$ 70$ \\
\hline Method: & $\begin{array}{l}\text { Smoothtalker software } \\
\text { D/A converter }\end{array}$ & $\begin{array}{l}\text { Votrax SC02 } \\
\text { IC chip }\end{array}$ & $\begin{array}{l}\text { GI IC chips } \\
\text { CTS256A-AL2 and SPO256-AL2 }\end{array}$ \\
\hline Interface: & $\begin{array}{l}\text { Printer port with } \\
\text { supplied software driver }\end{array}$ & $\begin{array}{l}\text { PC bus with supplied } \\
\text { software driver }\end{array}$ & $\begin{array}{l}\text { Serial port with } \\
\text { MS-DOS COM1 driver }\end{array}$ \\
\hline Installation: & $\begin{array}{l}\text { Plugs into printer port } \\
\text { and keyboard power }\end{array}$ & $\begin{array}{l}\text { Electronic assembly, } \\
\text { soldering, etc; } \\
\text { Plugs into PC bus }\end{array}$ & $\begin{array}{l}\text { Plugs into PC bus, } \\
\text { but only for power }\end{array}$ \\
\hline Symbols: & English and phonemes & English and phonemes & English only \\
\hline $\begin{array}{l}\text { Voicing } \\
\text { parameters: }\end{array}$ & 2 & 10 & none \\
\hline Optional PCM?: & yes & no & no \\
\hline $\begin{array}{l}\text { Phonemes and } \\
\text { allophones: }\end{array}$ & 34 & 64 & (English only) \\
\hline Speaker: & External, supplied & External, supplied & Internal PC speaker \\
\hline
\end{tabular}

